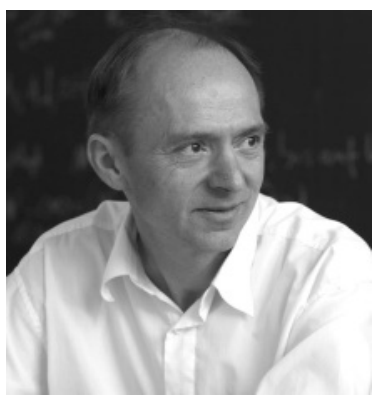

\author{
Juraj Hromkovič, \\ ETH Zürich, \\ E-Mail: \\ juraj.hromkovic@inf.ethz.ch
}

\title{
Bilden wir die Erfinderinnen, Gestalter und Entwicklerinnen digitaler Technologie aus und nicht nur ihre Konsumenten!
}

Kaum ein anderes Fach erlebte und erlebt einen so turbulenten Einzug in das allgemeine Bildungssystem wie die Informatik. Jedes Land schreibt seine eigene Geschichte und diese könnten kaum unterschiedlicher sein. Der erfolgreichste und am besten definierte Einzug der Informatik in das Bildungssystem erfolgte vor fast 50 Jahren in Russland und den meisten osteuropäischen Ländern. Ich selbst genoss als Gymnasiast einen soliden Informatikunterricht für die Dauer von vier Jahren (1973-1977), jeweils vier Stunden wöchentlich. Drei Programmiersprachen, Datenverwaltung mit Cobol und Algorithmen für das Sortieren, die Suche, lineare Algebra und numerische Mathematik standen damals im Mittelpunkt.

Während die Informatik im Osten ganz selbstverständlich als ein normales Fach etabliert wurde, gab es in den achtziger Jahren - zumeist über das Programmieren - in den USA und in Westeuropa erste Versuche, die Informatik in den Bildungssystemen zu verankern. Mitte der neunziger Jahre kam in diesen Ländern der Rückschlag aufgrund der naiven Ideologie, dass das Allerwichtigste das Erlernen des Umgangs mit dem Computer und mit der vorhandenen Software sei und dass ein eigenes Fach Informatik nur für Fachspezialisten geeignet wäre. Die Reduktion der Informatik auf oberflächliche Betriebsanweisungen und Computerführerscheine beschädigte das Image des jungen Faches enorm und somit auch das Innovationspotenzial der Wirtschaft. Es folgte eine vergleichbar starke Herabstufung der Informatik, als würde man den Unterricht in Physik und dem Fach Maschinenbau auf den Erwerb eines Führerscheins reduzieren. Der sogenannte Informations- und Kommunikationstechnik-Unterricht (IKT/ICT) wurde zum einzigen Schulfach, für das man keine Lehrerausbildung brauchte, es reichten wenige Kurswochen.

Rund zehn Jahre danach haben die meisten Länder diesen groben Fehler erkannt. Die USA, Großbritannien, Frankreich und Italien führten zum Beispiel schrittweise einen obligatorischen Informatikunterricht ein, und zwar mit klarer Trennung von der Vermittlung von Anwendungskompetenzen. Nur im deutschsprachigen Raum - mit kleinen Ausnahmen wie Bayern - tut man sich damit schwer. Die Ideologie des „Computerführerscheins" wurde durch die Ideologie der "Medienkunde" ersetzt. Zum Allerwichtigsten wurden dabei die Reflexionen über die Inhalte, die mittels der Informationstechnologie verbreitet werden. Statt diese Kommunikationsaspekte und die damit verbundenen ethischen Fragen mit den Sprachwissenschaften und der Ethik zu verbinden, bemühte man sich um die "Zwangsheirat" mit der Informatik. Als Reaktion auf das Manifest der deutschsprachigen Informatikerinnen und Informatiker für ein eigenständiges Fach Informatik ohne ICT und Medien aus Dagstuhl vom Winter 2015 folgte das „DagstuhlDreieck" der Medienwissenschaftler. Es betonte die Einheit der Medienwissenschaft, ICT und Informatik und schrieb der Medienkunde die führende Rolle zu. Das „Dagstuhl-Dreieck“ reduzierte die Informatik auf das Reflektieren über die Technologie; die Gestaltung und die Entwicklung der Informatik kommen dabei nicht vor. Überall, wo die Ideologie des „Dagstuhl-Dreiecks" zur Anwendung kam, ist es zu keinem nennenswerten Informatikunterricht gekommen. Das betrifft die Lehrmittel sowie die Aus- und Weiterbildung von Lehrpersonen.

Deswegen droht zum Beispiel in der Schweiz, der erneute Versuch, Informatik im Rahmen des Faches „Medien und Informatik" in der obligatorischen Schule einzuführen, zu scheitern. Die Informatik braucht wie alle etablierten Schulfächer ein Spiralcurriculum. Man muss ein gewisses Wissen und gewisse Kompetenzen zuerst festigen als Vorstufe für eine Vertiefung des Wissens. Anders als in der Medienkunde, die ein unabhängiges Thema nach dem anderen bearbeiten kann, ist dies in der Informatik nicht möglich, weil die einzelnen Wissensschritte aufeinander aufbauen.

https://doi.org/10.1007/s00287-019-01174-1 


\section{$\{$ EDITORIAL}

Dieses Sonderheft kommt deswegen heraus. Wir stellen uns die wichtigsten Fragen für den Einzug der Informatik in die allgemeine Bildung und versuchen, sie zu beantworten: "Was ist Informatik?", "Warum ist sie allgemeinbildend?", „,Welchen Mehrwert bringt die Informatik für die Schule?", „Wie fördert ein guter Informatikunterricht das Erreichen der grundlegenden Kompetenzen in anderen Fächern?", „Wie trägt der Informatikunterricht zum Verständnis der Welt und zur Vorbereitung auf die Berufe der Zukunft bei?", „Wie vermittelt man die wichtigsten Konzepte der Informatik in unterschiedlichen Altersstufen?" und "Wie stärkt der Informatikunterricht das Innovationspotenzial der Gesellschaft?"

Ich wünsche allen, insbesondere den Verantwortlichen und den Entscheidungsträgern und Entscheidungsträgerinnen, viel Vergnügen bei der Lektüre und lade alle ein, für das Schulfach Informatik die Lanze zu brechen. 


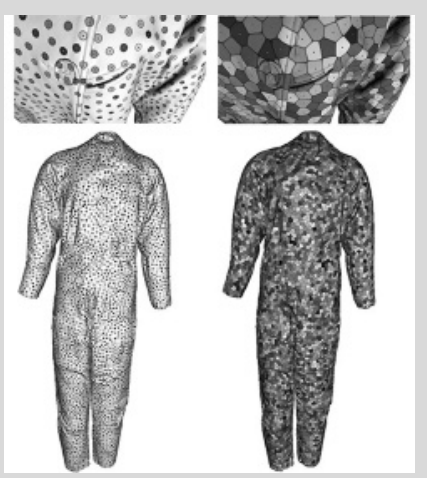

Illustration of the growth of natural tessellations (an alternative numerical model to Voronoi diagrams on surfaces) on the Earhart's Flight Suit (10M facets mesh, courtesy of the Smithsonian Institution) initialized with 10,000 random seeds, their corresponding cells are visualized in different colors. The close-up on the right side of the chest (top-row) reveals the small scale geometric complexities (pocket fold, button fold) that can successfully be processed with this computationally and memory efficient approach fully implemented on graphics hardware (GPU).

Rhaleb Zayer, Daniel Mlakar, Markus Steinberger, and Hans-Peter Seidel. 2018. Layered fields for natural tessellations on surfaces. ACM Trans. Graph. 37(6), Article 264 (December 2018). 